\title{
The $m$-colored composition poset
}

\author{
Brian Drake \\ Department of Mathematics \\ Brandeis University \\ Waltham, MA 02454 \\ bdrake@brandeis.edu \\ T. Kyle Petersen \\ Department of Mathematics \\ University of Michigan \\ Ann Arbor, MI 48109 \\ tkpeters@umich.edu
}

Submitted: Oct 4, 2006; Accepted: Feb 13, 2007; Published: Feb 27, 2007

Mathematics Subject Classification: 06A07, 05A99, 52B22

\begin{abstract}
We define a partial order on colored compositions with many properties analogous to Young's lattice. We show that saturated chains correspond to colored permutations, and that covering relations correspond to a Pieri-type rule for colored quasi-symmetric functions. We also show that the poset is CL-shellable. In the case of a single color, we recover the subword order on binary words.
\end{abstract}

\section{Introduction}

A partition $\lambda=\left(\lambda_{1}, \lambda_{2}, \ldots\right)$ of $n$, denoted $\lambda \vdash n$, is a sequence of nonnegative integers $\lambda_{1} \geq \lambda_{2} \geq \cdots \geq 0$ such that $\sum \lambda_{i}=n$. The set of all partitions of all integers $n \geq 0$ forms a lattice under the partial order given by inclusion of Young diagrams: $\lambda \leq \mu$ if $\lambda_{i} \leq \mu_{i}$ for all $i$. This lattice, which is called Young's lattice, $Y$, has several remarkable properties, including the following list.

Y1. $Y$ is a graded poset, where a partition $\lambda \vdash n$ has rank $n$.

Y2. The number of saturated chains from the minimal partition $\emptyset$ to $\lambda$ is the number $f^{\lambda}$ of Young tableaux of shape $\lambda$.

Y3. The number of saturated chains from $\emptyset$ to rank $n$ is the number of involutions in the symmetric group $\mathfrak{S}_{n}$. 
Y4. Let $s_{\lambda}$ denote a Schur function. Pieri's rule [7] gives

$$
s_{1} s_{\lambda}=\sum_{\lambda \prec \mu} s_{\mu}
$$

where $\lambda \prec \mu$ means that $\mu$ covers $\lambda$ in $Y$.

Y5. Since $Y$ is in fact a distributive lattice, every interval $[\lambda, \mu]$ is EL-shellable and hence Cohen-Macaulay.

For each of the properties Y1-Y5, there is an analogous property for the poset of $m$-colored compositions.

Recall that a composition $\alpha=\left(\alpha_{1}, \alpha_{2}, \ldots, \alpha_{k}\right)$ is an ordered tuple of positive integers, called the parts of $\alpha$. We write $l(\alpha)=k$ for the number of parts of $\alpha$. If the sum of the parts of $\alpha$ is $n$, i.e., $|\alpha|:=\alpha_{1}+\alpha_{2}+\cdots+\alpha_{k}=n$, then we say $\alpha$ is a composition of $n$, written $\alpha \models n$.

Fix a positive integer $m$ and any primitive $m$-th root of unity, $\omega$. An $m$-colored composition is an ordered tuple of colored positive integers, say $\alpha=\left(\varepsilon_{1} \alpha_{1}, \varepsilon_{2} \alpha_{2}, \ldots, \varepsilon_{k} \alpha_{k}\right)$, where the $\alpha_{s}$ are positive integers and $\varepsilon_{s}=\omega^{i_{s}}, 0 \leq i_{s} \leq m-1$. We say the part $\varepsilon_{s} \alpha_{s}$ has color $\varepsilon_{s}$, and we write $\alpha \models_{m} n$ if $|\alpha|:=\alpha_{1}+\alpha_{2}+\cdots+\alpha_{k}=n$. For example, if $m=3$, then $\alpha=\left(\omega 2,1, \omega^{2} 1,3\right)$ is a 3 -colored composition of $2+1+1+3=7$.

Note that there are $m^{k}$ ways to color any ordinary composition of $n$ with $k$ parts, leading us to conclude that there are

$$
\sum_{k=1}^{n}\left(\begin{array}{l}
n-1 \\
k-1
\end{array}\right) m^{k}=m(m+1)^{n-1}
$$

$m$-colored compositions of $n$ (so if $m=1$, we have $2^{n-1}$ ordinary compositions). Let $\operatorname{Comp}^{(m)}(n)$ denote the set of all $m$-colored compositions of $n$, and define

$$
\mathcal{C}^{(m)}:=\bigcup_{n \geq 0} \operatorname{Comp}^{(m)}(n)
$$

where $\emptyset$ is the unique composition of 0 .

We can define a partial order on $\mathcal{C}^{(m)}$ via covering relations as follows. We say that $\beta$ covers $\alpha$ if, for some $j$, we can write $\beta$ as:

1. $\left(\varepsilon_{1} \alpha_{1}, \ldots, \varepsilon_{j-1} \alpha_{j-1}, \varepsilon_{j}\left(\alpha_{j}+1\right), \varepsilon_{j+1} \alpha_{j+1}, \ldots, \varepsilon_{k} \alpha_{k}\right)$,

2. $\left(\varepsilon_{1} \alpha_{1}, \ldots, \varepsilon_{j-1} \alpha_{j-1}, \varepsilon_{j}(h+1), \varepsilon_{j}\left(\alpha_{j}-h\right), \varepsilon_{j+1} \alpha_{j+1}, \ldots, \varepsilon_{k} \alpha_{k}\right)$ for some $0 \leq h \leq \alpha_{j}-1$, or

3. $\left(\varepsilon_{1} \alpha_{1}, \ldots, \varepsilon_{j-1} \alpha_{j-1}, \varepsilon_{j} h, \varepsilon^{\prime} 1, \varepsilon_{j}\left(\alpha_{j}-h\right), \varepsilon_{j+1} \alpha_{j+1}, \ldots, \varepsilon_{k} \alpha_{k}\right)$ where $\varepsilon^{\prime} \neq \varepsilon_{j}$ and $0 \leq$ $h \leq \alpha_{j}-1$, with the understanding that we will ignore parts of size 0 . 
With relations (1) and (2) we preserve the color, adding 1 to a part, or adding 1 to a part and splitting that part into two parts. Relation (3) handles the case where the color of the "1" we add differs from where we try to add it. Notice that it is immediate from these covering relations that $\mathcal{C}^{(m)}$ is a graded poset with level $n$ consisting of all $m$-compositions of $n$. This property is analogous to property Y1 of Young's lattice. See Figure 1 for the first four levels of the 2-colored composition poset.

In the case of a single color, the poset $\mathcal{C}^{(1)}$ is already known with a different description. The covering relations simplify so that if $\beta$ covers $\alpha$, then for some $j$ we can write $\beta$ as:

1. $\left(\alpha_{1}, \ldots, \alpha_{j-1}, \alpha_{j}+1, \alpha_{j+1}, \ldots, \alpha_{k}\right)$, or

2. $\left(\alpha_{1}, \ldots, \alpha_{j-1}, h+1, \alpha_{j}-h, \alpha_{j+1}, \ldots, \alpha_{k}\right)$ for some $0 \leq h \leq \alpha_{j}-1$.

Here we recall a well-known bijection from nonempty compositions to binary words. Let $A^{*}$ denote all words in a two letter alphabet $A=\{a, b\}$. Let $\alpha=\left(\alpha_{1}, \alpha_{2}, \ldots, \alpha_{k}\right)$ be a composition. We map $\alpha$ to the word $\phi\left(\alpha_{1}\right) \phi\left(\alpha_{2}\right) \cdots \phi\left(\alpha_{k}\right)$, where $\phi$ is defined on positive integers by $\phi(h)=b^{h-1} a$, and then remove the final $a$. For example, $(3,1,1,2,1) \mapsto$ bbaaaba. It is not difficult to see that this is a set bijection. Also, covering relations of type (1) correspond to inserting or deleting a $b$, and covering relations of type (2) correspond to inserting or removing an $a$. These are exactly the covering relations for the subword order on binary words [2], hence this map is a poset isomorphism between $\mathcal{C}^{(1)}$ (with the minimal element removed), and the subword order on binary words.

The subword order has been studied, and analogues of Y1-Y5 are known for a twoletter alphabet. Path counting properties similar to Y2 and Y3 can easily be derived from Fomin's theory of dual graded graphs [4]. Another approach is the "hypoplactic correspondence" of Krob and Thibon [5]. An analogue of Y4 is a Pieri-type rule for multiplying fundamental quasi-symmetric functions. This can be found, for example, by setting $\alpha=1$ in [7, Exer. 7.93]. The subword order on a finite alphabet is CL-shellable, analogous to Y5, as shown by Björner [2].

Remark 1.1. All of these links were outlined in a wonderful preprint by Björner and Stanley which, sadly, is no longer available on the ArXiv. This paper was meant to be a straightforward extension of their work on the case $\mathcal{C}^{(1)}$. In any case, we owe Björner and Stanley thanks for the general framework of this paper and for bringing the subject to our attention.

In section 2 we discuss colored permutations, their color-descent compositions, and chains in $\mathcal{C}^{(m)}$. In section 3 we present Poirier's colored quasisymmetric functions [6] and show that $\mathcal{C}^{(m)}$ gives a Pieri-type rule for multiplying a fundamental basis. We define a CL-labeling in section 4 and use this to calculate the Möbius function of lower intervals. Section 5 contains the proof that this labeling is a CL-labeling.

\section{Colored permutations and descent sets}

Compositions can be used to encode descent classes of ordinary permutations in the following way. Recall that a descent of a permutation $w \in \mathfrak{S}_{n}$ is a position $i$ such that 


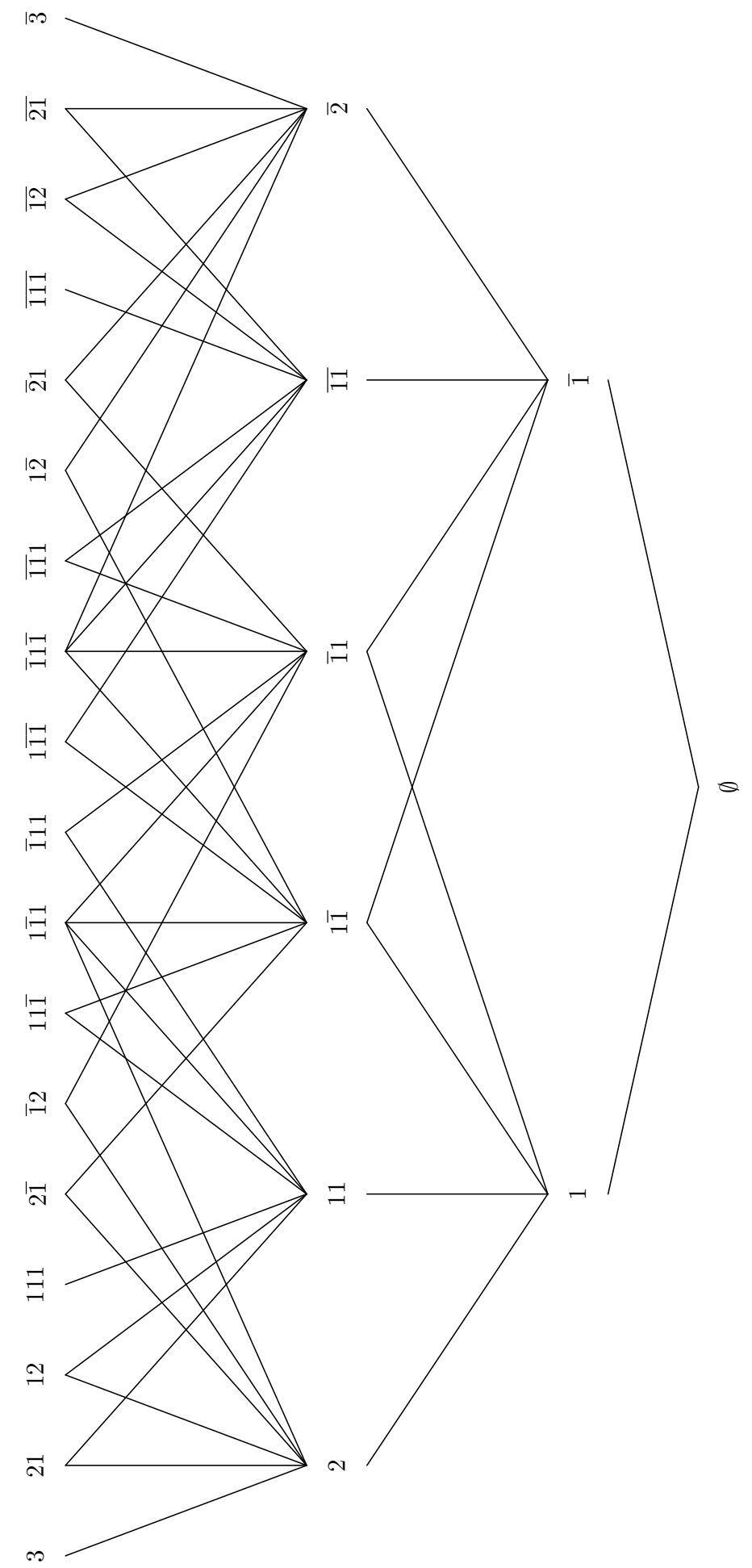

Figure 1: The first four levels of the 2-colored composition poset. 
$w_{i}>w_{i+1}$, and that an increasing run (of length $r$ ) of a permutation $w$ is a maximal subword of consecutive letters $w_{i+1} w_{i+2} \cdots w_{i+r}$ such that $w_{i+1}<w_{i+2}<\cdots<w_{i+r}$. By maximality, we have that if $w_{i+1} w_{i+2} \cdots w_{i+r}$ is an increasing run, then $i$ is a descent of $w$ (if $i \neq 0$ ), and $i+r$ is a descent of $w$ (if $i+r \neq n$ ). For any permutation $w \in \mathfrak{S}_{n}$ define the descent composition, $\mathrm{C}(w)$, to be the ordered tuple listing from left to right the lengths of the increasing runs of $w$. If $\mathrm{C}(w)=\left(\alpha_{1}, \alpha_{2}, \ldots, \alpha_{k}\right)$, we can recover the descent set of $w$ :

$$
\operatorname{Des}(w):=\left\{i: w_{i}>w_{i+1}\right\}=\left\{\alpha_{1}, \alpha_{1}+\alpha_{2}, \ldots, \alpha_{1}+\alpha_{2}+\cdots+\alpha_{k-1}\right\} .
$$

For example, the permutation $w=345261$ has $\mathrm{C}(w)=(3,2,1)$ and $\operatorname{Des}(w)=\{3,5\}$. We now define colored permutations and colored descent compositions.

Loosely speaking, $m$-colored permutations are permutations where each of the elements permuted are given one of $m$ "colors." If $\omega$ is any primitive $m$-th root of unity, then

$$
\omega 3 \omega 21 \omega^{3} 4
$$

is an example of a colored permutation. We can think of building colored permutations by taking an ordinary permutation and then arbitrarily assigning colors to the letters, so we see that there are $m^{n} n ! m$-colored permutations of $[n]:=\{1,2, \ldots, n\}$.

Strictly speaking, $m$-colored permutations are elements of the wreath product $C_{m} \imath \mathfrak{S}_{n}$, where $C_{m}=\left\{1, \omega, \ldots, \omega^{m-1}\right\}$ is the cyclic group of order $m$. We write an element $u=$ $u_{1} u_{2} \cdots u_{n} \in C_{m} \succ \mathfrak{S}_{n}$ as a word in the alphabet

$$
[n] \times C_{m}:=\left\{1, \omega 1, \ldots, \omega^{m-1} 1,2, \omega 2, \ldots, \omega^{m-1} 2, \ldots, n, \omega n, \ldots, \omega^{m-1} n\right\},
$$

such that $|u|=\left|u_{1}\right|\left|u_{2}\right| \cdots\left|u_{n}\right|$ is an ordinary permutation in $\mathfrak{S}_{n}$. We say $\varepsilon_{i}=u_{i} /\left|u_{i}\right|$ is the color of $u_{i}$.

For any $u \in C_{m} \imath \mathfrak{S}_{n}$, we can write $u=v_{1} v_{2} \cdots v_{k}$ so that each $v_{i}$ is a word in which all the letters have the same color, $\varepsilon_{i}^{\prime}$, and no two consecutive colors are the same: $\varepsilon_{i}^{\prime} \neq \varepsilon_{i+1}^{\prime}$, $i=1,2, \ldots, k-1$. Then we define the color composition of $u$,

$$
\operatorname{Col}(u):=\left(\varepsilon_{1}^{\prime} \alpha_{1}^{\prime}, \varepsilon_{2}^{\prime} \alpha_{2}^{\prime}, \ldots, \varepsilon_{k}^{\prime} \alpha_{k}^{\prime}\right),
$$

where $\alpha_{s}^{\prime}$ denotes the number of letters in $v_{s}$. Now suppose an $m$-colored permutation $u$ has color composition $\operatorname{Col}(u)=\left(\varepsilon_{1}^{\prime} \alpha_{1}^{\prime}, \varepsilon_{2}^{\prime} \alpha_{2}^{\prime}, \ldots, \varepsilon_{k}^{\prime} \alpha_{k}^{\prime}\right)$. Then the colored descent composition

$$
\mathrm{C}^{(m)}(u):=\left(\varepsilon_{1} \alpha_{1}, \varepsilon_{2} \alpha_{2}, \ldots, \varepsilon_{l} \alpha_{l}\right),
$$

is the refinement of $\operatorname{Col}(u)$ where we replace part $\varepsilon_{i}^{\prime} \alpha_{i}^{\prime}$ with $\varepsilon_{i}^{\prime} \mathrm{C}\left(\left|v_{i}\right|\right)$, where $\mathrm{C}$ is the ordinary descent composition, and we view $\left|v_{i}\right|$ as an ordinary permutation of distinct letters.

More intuitively, the colored descent composition $\mathrm{C}^{(m)}(u)$ is the ordered tuple listing the lengths of increasing runs of $u$ with constant color, where we record not only the length of such a run, but also its color. An example should cement the notion. If we have two colors (indicated with a bar), let

$$
u=1 \overline{2} \overline{3} 4 \overline{8} \overline{5} 76 .
$$


Then the color composition is $\operatorname{Col}(u)=(1, \overline{2}, 1, \overline{2}, 2)$, and

$$
\mathrm{C}^{(m)}(u)=(1, \overline{2}, 1, \overline{1}, \overline{1}, 1,1) .
$$

For any $\alpha \in \operatorname{Comp}^{(m)}(n)$, a saturated chain from $\emptyset$ to $\alpha$ is a sequence of compositions

$$
\emptyset=\alpha^{0} \prec \alpha^{1} \prec \cdots \prec \alpha^{n}=\alpha,
$$

where $\prec$ denotes a cover relation in $\mathcal{C}^{(m)}$, and therefore $\alpha^{i} \in \operatorname{Comp}^{(m)}(i)$. Now, given any $u \in C_{m} \prec \mathfrak{S}_{n}$, let $u[i]$ denote the restriction of $u$ to letters in $C_{m} \times[i]$. For example, if $u=\overline{2} 17 \overline{6} \overline{3} \overline{4} 58$, then $u[5]=\overline{2} 1 \overline{3} \overline{4} 5$. We then define the sequence

$$
\mathfrak{m}(u):=\left(\mathrm{C}^{(m)}(u[1]), \ldots, \mathrm{C}^{(m)}(u[n])\right),
$$

so that $\mathrm{C}^{(m)}(u[i]) \in \operatorname{Comp}^{(m)}(i)$. Using the same example $u=\overline{2} 17 \overline{6} \overline{3} \overline{4} 58$, we have

$$
\mathfrak{m}(u)=(1, \overline{1} 1, \overline{1} 1 \overline{1}, \overline{1} 1 \overline{2}, \overline{1} 1 \overline{2} 1, \overline{1} 1 \overline{1} \overline{2} 1, \overline{1} 2 \overline{1} \overline{2} 1, \overline{1} 2 \overline{1} \overline{2} 2) .
$$

Theorem 2.1. The map $\mathfrak{m}$ is a bijection from $C_{m} \prec \mathfrak{S}_{n}$ to saturated chains from $\emptyset$ to $\alpha$, where $\alpha$ ranges over all colored compositions in $\operatorname{Comp}^{(m)}(n)$.

Proof. For any colored permutation $u \in C_{m} \prec \mathfrak{S}_{n}$ define, for all $0 \leq i \leq n$ and all $0 \leq j \leq m-1$,

$$
u_{(i, j)}:=u_{1} \cdots u_{i} \omega^{j}(n+1) u_{i+1} \cdots u_{n} .
$$

In other words, the $u_{(i, j)}$ are all those permutations $w$ in $C_{m} \prec \mathfrak{S}_{n+1}$ such that $w[n]=u$. We will show that the compositions $\mathrm{C}^{(m)}\left(u_{(i, j)}\right)$ are all distinct and moreover that they are precisely those compositions in $\operatorname{Comp}^{(m)}(n+1)$ that cover $\mathrm{C}^{(m)}(u)$.

Suppose $\mathrm{C}^{(m)}(u)=\left(\varepsilon_{1} \alpha_{1}, \ldots, \varepsilon_{k} \alpha_{k}\right)$, and let $b_{s}=\alpha_{1}+\cdots+\alpha_{s}$, with the convention that $b_{0}=0$. For any fixed $j=0,1, \ldots, m-1$, we have two cases, corresponding to cover relations of type (1) or type (3):

$$
\mathrm{C}^{(m)}\left(u_{\left(b_{s}, j\right)}\right)= \begin{cases}\left(\varepsilon_{1} \alpha_{1}, \ldots, \varepsilon_{s}\left(\alpha_{s}+1\right), \ldots, \varepsilon_{k} \alpha_{k}\right) & \text { if } \varepsilon_{s}=\omega^{j} \\ \left(\varepsilon_{1} \alpha_{1}, \ldots, \varepsilon_{s} \alpha_{s}, \omega^{j} 1, \ldots, \varepsilon_{k} \alpha_{k}\right) & \text { otherwise }\end{cases}
$$

All these compositions, over $s=0, \ldots, k, j=0, \ldots, m-1$, are distinct and cover $\mathrm{C}^{(m)}(u)$. To consider the other cases, suppose $i$ is not of the form $\alpha_{1}+\cdots+\alpha_{s}$. Then it can be written as $i=\alpha_{1}+\cdots+\alpha_{s}+h$, where $0 \leq s \leq k$ and $1 \leq h \leq \alpha_{s+1}-1$ (if $s=0$, then $i=h)$. Again we have two cases, corresponding to cover relations of type (2) or type (3):

$$
\mathrm{C}^{(m)}\left(u_{(i, j)}\right)= \begin{cases}\left(\varepsilon_{1} \alpha_{1}, \ldots, \varepsilon_{s+1}(1+h), \varepsilon_{s+1}\left(\alpha_{s+1}-h\right), \ldots, \varepsilon_{k} \alpha_{k}\right) & \text { if } \varepsilon_{s+1}=\omega^{j}, \\ \left(\varepsilon_{1} \alpha_{1}, \ldots, \varepsilon_{s+1} h, \omega^{j} 1, \varepsilon_{s+1}\left(\alpha_{s+1}-h\right), \ldots, \varepsilon_{k} \alpha_{k}\right) & \text { otherwise. }\end{cases}
$$

These cases are again distinct and provide the remaining covers for $\mathrm{C}^{(m)}(u)$.

Theorem 2.1 yields several easy corollaries. The first is analogous to property Y2 of Young's lattice; the second corresponds to Y3. 
Corollary 2.2. The number of saturated chains from $\emptyset$ to $\alpha$ in $\mathcal{C}^{(m)}$ is equal to the number $f_{n}^{(m)}(\alpha)$ of $m$-colored permutations $w$ with colored descent composition $\alpha$.

Corollary 2.3. The total number of saturated chains from $\emptyset$ to rank $n$ is equal to the number of $m$-colored permutations of $[n]$,

$$
\sum_{\alpha \in \mathrm{Comp}^{(m)}(n)} f_{n}^{(m)}(\alpha)=m^{n} n ! .
$$

Corollary 2.4. The number of $m$-colored compositions $\beta \in \operatorname{Comp}^{(m)}(n+1)$ covering $\alpha \in \operatorname{Comp}^{(m)}(n)$ is $m(n+1)$.

\section{Colored quasisymmetric functions}

One key use for compositions is as an indexing set for quasisymmetric functions. Similarly, there exist colored quasisymmetric functions (due to Poirier [6]) that use colored compositions as indices. Both these situations are analogous to how partitions index symmetric functions.

Recall ([7], ch. 7.19) that a quasisymmetric function is a formal series

$$
Q\left(x_{1}, x_{2}, \ldots\right) \in \mathbb{Z}\left[\left[x_{1}, x_{2}, \ldots\right]\right]
$$

of bounded degree such that for any composition $\alpha=\left(\alpha_{1}, \alpha_{2}, \ldots, \alpha_{k}\right)$, the coefficient of $x_{i_{1}}^{\alpha_{1}} x_{i_{2}}^{\alpha_{2}} \cdots x_{i_{k}}^{\alpha_{k}}$ with $i_{1}<i_{2}<\cdots<i_{k}$ is the same as the coefficient of $x_{1}^{\alpha_{1}} x_{2}^{\alpha_{2}} \cdots x_{k}^{\alpha_{k}}$. One natural basis for the quasisymmetric functions homogeneous of degree $n$ is given by the fundamental quasisymmetric functions, $L_{\alpha}$, where $\alpha$ ranges over all of $\operatorname{Comp}(n)$. If $\alpha=\left(\alpha_{1}, \ldots, \alpha_{k}\right) \models n$, then define

$$
L_{\alpha}:=\sum x_{i_{1}} \cdots x_{i_{n}}
$$

where the sum is taken over all $i_{1} \leq i_{2} \leq \cdots \leq i_{n}$ with $i_{s}<i_{s+1}$ if $s=\alpha_{1}+\cdots+\alpha_{r}$ for some $r$. For example,

$$
L_{21}=\sum_{i \leq j<k} x_{i} x_{j} x_{k}
$$

Colored quasisymmetric functions are simply a generalization of quasisymmetric functions to an alphabet with several colors for its letters. For fixed $m$, we consider formal series in the alphabet

$$
X^{(m)}:=\left\{x_{1,0}, x_{1,1}, \ldots, x_{1, m-1}, x_{2,0}, x_{2,1} \ldots, x_{2, m-1}, \ldots\right\},
$$

(so the second subscript corresponds to color) with the same quasisymmetric property. Namely, an $m$-colored quasisymmetric function $Q\left(X^{(m)}\right)$ is a formal series of bounded degree such that for any $m$-colored composition $\alpha=\left(\omega^{j_{1}} \alpha_{1}, \ldots, \omega^{j_{k}} \alpha_{k}\right)$, the coefficient of $x_{i_{1}, j_{1}}^{\alpha_{1}} x_{i_{2}, j_{2}}^{\alpha_{2}} \cdots x_{i_{k}, j_{k}}^{\alpha_{k}}$ with $\left(i_{1}, j_{1}\right)<\left(i_{2}, j_{2}\right)<\cdots<\left(i_{k}, j_{k}\right)$ (in lexicographic order) is the 
same as the coefficient of $x_{1, j_{1}}^{\alpha_{1}} x_{2, j_{2}}^{\alpha_{2}} \cdots x_{k, j_{k}}^{\alpha_{k}}$. Intuitively, the letters are colored the same as the parts of $\alpha$. The m-colored fundamental quasisymmetric functions are defined as follows. First, if $s=\alpha_{1}+\cdots+\alpha_{r}+h, 1 \leq h \leq \alpha_{r+1}$, then define $j_{s}^{\prime}=j_{r+1}$, the color of part $\alpha_{r+1}$. Then,

$$
L_{\alpha}^{(m)}:=\sum x_{i_{1}, j_{1}^{\prime}} \cdots x_{i_{n}, j_{n}^{\prime}},
$$

where the sum is taken over all $i_{1} \leq i_{2} \leq \cdots \leq i_{n}$ with $i_{s}<i_{s+1}$ if both $j_{s}^{\prime} \geq j_{s+1}^{\prime}$ and $s=\alpha_{1}+\cdots+\alpha_{r}$ for some $r$. For example,

$$
L_{1 \overline{2} \overline{1}}^{(2)}=\sum_{i \leq j \leq k<l} x_{i} y_{j} y_{k} y_{l} \quad \text { and } \quad L_{2 \overline{1} \overline{2}}^{(3)}=\sum_{i \leq j \leq k<l \leq m} x_{i} x_{j} z_{k} y_{l} y_{m}
$$

As in the ordinary case, the $L_{\alpha}^{(m)}$, where $\alpha$ ranges over $\operatorname{Comp}^{(m)}(n)$, give a basis for the $m$-colored quasisymmetric functions homogeneous of degree $n$.

There is a nice formula for multiplying colored quasisymmetric functions in the fundamental basis. Let $u \in C_{m} \geq \mathfrak{S}_{n}$ and let $v$ be an $m$-colored permutation of the set $\{n+1, n+2, \ldots, n+r\}$. Let $\alpha=\mathrm{C}^{(m)}(u)$ and $\beta=\mathrm{C}^{(m)}(v)$. Then we have

$$
L_{\alpha}^{(m)} L_{\beta}^{(m)}=\sum_{w} L_{\mathrm{C}^{(m)}(w)}^{(m)}
$$

where the sum is taken over all shuffles $w$ of $u$ and $v$, i.e., all colored permutations $w \in C_{m}\left\langle\mathfrak{S}_{n+r}\right.$ such that $w[n]=u$ and $w$ restricted to $\{n+1, n+2, \ldots, n+r\}$ is $v$.

If $r=1$, then we see that the shuffles of $u$ and $v=\omega^{j}(n+1)$ are precisely those permutations $u_{(i, j)}$ from the proof of Theorem 2.1. Applying the multiplication rule, and summing over all $j$, we have a Pieri-type rule analogous to property Y4 of Young's lattice.

Proposition 3.1. We have:

$$
\left(L_{1}^{(m)}+L_{\omega 1}^{(m)}+\cdots+L_{\omega^{m-1} 1}^{(m)}\right) L_{\alpha}^{(m)}=\sum_{\alpha \prec \beta} L_{\beta}^{(m)} .
$$

In fact, we could have used Proposition 3.1 to define the poset $\mathcal{C}^{(m)}$ in the first place. At the least, it is a good justification for the study of $\mathcal{C}^{(m)}$.

Repeated application of the proposition gives the formula

$$
\left(L_{1}^{(m)}+L_{\omega 1}^{(m)}+\cdots+L_{\omega^{m-1} 1}^{(m)}\right)^{n}=\sum_{\alpha \in \operatorname{Comp}^{(m)}(n)} f_{n}^{(m)}(\alpha) L_{\alpha}^{(m)},
$$

where $f_{n}^{(m)}$ is the number of $m$-colored permutations with colored descent composition $\alpha$. This equation is equivalent to Corollary 2.2, and analogous to the following formula for Schur functions (see [7]) that corresponds to property Y2 of Young's lattice:

$$
s_{1}^{n}=\sum_{\lambda \vdash n} f^{\lambda} s_{\lambda},
$$

where $f^{\lambda}$ is the number of Young tableaux of shape $\lambda$. 


\section{Shellability and Möbius function}

In this section we show that $\mathcal{C}^{(m)}$ is CL-shellable by giving an explicit dual CL-labeling. See [3] for an introduction to CL-shellable posets. We use a model of removing colored balls from urns to define our labeling on downward maximal chains. Given a colored composition of $n, \alpha=\left(\varepsilon_{1} \alpha_{1}, \ldots, \varepsilon_{k} \alpha_{k}\right)$, we picture $k$ urns next to each other, labeled $U_{1}, U_{2}, \ldots, U_{k}$ from left to right. In urn $U_{i}$ we start with $\alpha_{i}$ balls of color $\varepsilon_{i}$, for a total of $n$ balls. Moving down along a maximal chain, we remove a ball from an urn for each covering relation, and possibly move some balls from one urn to another. There are three different types of moves, which we now describe. After some number of steps, suppose that $U_{i}$ is a nonempty urn, $U_{h}$ is the first nonempty urn on its left, and $U_{j}$ is the first nonempty urn on its right. Let $\beta_{i}, \beta_{h}, \beta_{j}$ be the number of balls in the corresponding urns and let $\varepsilon_{i}, \varepsilon_{h}, \varepsilon_{j}$ be the colors of those balls. The three possible moves are:

1. If $\beta_{i} \geq 2$, or if $\varepsilon_{h} \neq \varepsilon_{i}$, or if $U_{i}$ is the first nonempty urn, then remove a ball from $\operatorname{urn} U_{i}$.

2. If $\beta_{i}=1$ and $\varepsilon_{h}=\varepsilon_{j} \neq \varepsilon_{i}$, then remove the ball from $U_{i}$ and place all the balls from $U_{h}$ and $U_{j}$ into $U_{i}$.

3. If $\beta_{i} \geq 2$ and $\varepsilon_{j}=\varepsilon_{i}$, then move all balls from $U_{j}$ to $U_{i}$ and remove a ball from $U_{i}$.

After any number of moves, we may associate the distribution of colored balls in urns with an element of $\mathcal{C}^{(m)}$. The different urns represent the parts of the composition, the number of balls in an urn is the size of that part, and the color of the balls is the color of the part. Here we ignore parts of size 0 . Notice that the color of a part is well defined, since none of the moves allows balls of different colors to be combined in a single urn. It is an easy exercise to check that each covering relation in $\mathcal{C}^{(m)}$ corresponds to one of these three possible moves for some urn, and furthermore that the urn and type of move are unique.

Let $[\emptyset, \alpha]$ be an interval in $\mathcal{C}^{(m)},|\alpha|=n$. We will now define a labeling $\lambda(c)=\left(\lambda_{1}(c)\right.$, $\left.\lambda_{2}(c), \ldots, \lambda_{n}(c)\right)$ for a maximal chain

$$
c=\left(\alpha=\alpha^{0} \succ \alpha^{1} \succ \cdots \succ \alpha^{n}=\emptyset\right) .
$$

Our set of labels is $\mathbb{N} \times\{1,2,3\}$, totally ordered with the lexicographic order. For each covering relation $\alpha^{r-1} \succ \alpha^{r}$ we have a unique urn and type of move that takes the distribution of balls in urns for $\alpha^{r-1}$ to the distribution for $\alpha^{r}$. Suppose that move is of type $t$, and removes a ball from urn $U_{i}$. Then we define the label $\lambda_{r}(c)=(i, t)$.

Notice that with labels defined on maximal chains in lower intervals $[\emptyset, \alpha]$, there is an induced labeling defined on maximal chains in arbitrary intervals $[\beta, \alpha]$. As an example, consider the following two maximal chains in [3, 22112]:

$$
\begin{aligned}
c_{0} & =(22 \overline{1} 2 \succ 12 \overline{1} 2 \succ 2 \overline{1} 2 \succ 1 \overline{1} 2 \succ 3) \\
c & =(22 \overline{1} 2 \succ 21 \overline{1} 2 \succ 2 \overline{1} 2 \succ 22 \succ 3)
\end{aligned}
$$


They are labeled $\lambda\left(c_{0}\right)=((1,1),(1,1),(2,1),(3,2))$ and $\lambda(c)=((2,1),(1,3),(3,1),(1,3))$. Pictured as colored balls and urns, we have:

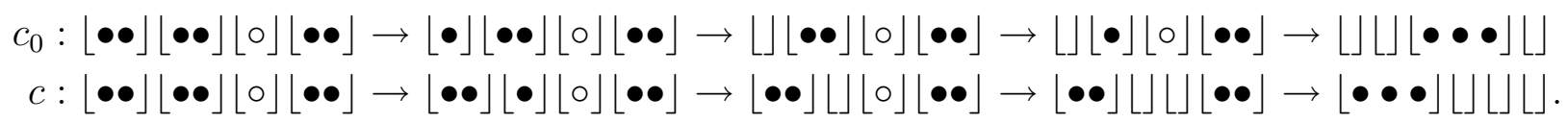

In fact, the chain $c_{0}$ above is lexicographically minimal and has the only increasing label.

By proving that this labeling is in fact a CL-labeling, we obtain our analog of property Y5 of Young's lattice. The proof is given in section 5.

Theorem 4.1. Intervals in $\mathcal{C}^{(m)}$ are dual $C L$-shellable and hence Cohen-Macaulay.

Now we calculate the Möbius function of lower intervals. As always, we must have $\mu_{\mathcal{C}^{(m)}}(\emptyset, \emptyset)=1$. For $\alpha \neq \emptyset$, we have the following.

\section{Proposition 4.2.}

$$
\mu_{\mathcal{C}^{(m)}}(\emptyset, \alpha)= \begin{cases}(-1)^{|\alpha|} & \text { if } \alpha=\left(\varepsilon_{1} 1, \varepsilon_{2} 1, \ldots, \varepsilon_{|\alpha|} 1\right) \\ & \text { for some colors } \varepsilon_{1} \neq \varepsilon_{2} \neq \cdots \neq \varepsilon_{|\alpha|}, \\ 0 & \text { otherwise. }\end{cases}
$$

Proof. We make use of the combinatorial description of the Möbius function for a graded poset with a CL-labeling, given in [3]. That is, the Möbius function of an interval is -1 to the length of the interval, times the number of maximal chains with a strictly decreasing label.

Suppose that $\alpha=\left(\varepsilon_{1} 1, \varepsilon_{2} 1, \ldots, \varepsilon_{|\alpha|} 1\right)$, with $\varepsilon_{1} \neq \varepsilon_{2} \neq \cdots \neq \varepsilon_{|\alpha|}$. Then there is a unique chain with a strictly decreasing label, obtained by removing the balls from right to left using only type $(1)$ moves. Therefore $\mu_{\mathcal{C}^{(m)}}(\emptyset, \alpha)=(-1)^{|\alpha|}$.

Now suppose that $\alpha$ has a part $i$ of size 2 or greater. We want to show that there is no chain in $[\emptyset, \alpha]$ with a strictly decreasing label. Any chain that makes a type (1) move from the same urn twice will have a repeated label. The only way to remove the balls from urn $i$ and possibly have a decreasing label is to remove at most one ball with a type (1) move, and then move all the balls to an urn on the left with a type (2) or (3) move. But in the new urn we have at least two balls, and the process repeats. At some point we must have an urn with at least two balls and no way to make a type (2) or (3) move. Then we must use two type (1) moves for the same urn, so the chain label cannot be strictly decreasing.

Finally, suppose that $\alpha$ has parts $\alpha_{i}$ and $\alpha_{i+1}$ of size 1 and the same color. The only legal way to remove the balls from the corresponding urns is to remove the left one first, creating an increase in the chain label.

Note that for $\alpha \models_{m} n$ with $\mu_{\mathcal{C}^{(m)}}(\emptyset, \alpha) \neq 0$, there are $m$ choices for the color of the first part, and $m-1$ choices for the color of each succeeding part. Hence there are $m(m-1)^{n-1}$ 
compositions $\alpha \models_{m} n$ with $\mu_{\mathcal{C}^{(m)}}(\emptyset, \alpha) \neq 0$. An elementary calculation gives the following generating function.

$$
\sum_{\alpha \in \mathcal{C}^{(m)}} \mu_{\mathcal{C}^{(m)}}(\emptyset, \alpha) t^{|\alpha|}=\frac{1+t}{1-(m-1) t} .
$$

Although the Möbius function of lower intervals is either $-1,0$, or 1 , this is not the case for arbitrary intervals. Proposition 4.3 provides an example. We would like to have an explicit formula for the Möbius function of an arbitrary interval (perhaps in terms of counting "embeddings" as in [2]), but such a formula has eluded us so far.

Proposition 4.3. Let $\omega_{n}=(1, \overline{1}, 1, \overline{1}, \ldots, 1)$ with $\left|\omega_{n}\right|=2 n-1$. Then $\mu_{\mathcal{C}^{(m)}}\left((1), \omega_{n}\right)=$ $2^{n}-1$.

Proof. By the theory of CL-labelings, $\mu_{\mathcal{C}^{(m)}}\left((1), \omega_{n}\right)$ counts strictly decreasing chains. It is not difficult to see that strictly decreasing chains in the interval $\left[(1), \omega_{n}\right]$ are obtained with only type (1) and (2) moves. Immediately after a type (2) move, a type (1) move must be made in the same urn.

In such a chain, we will call a particular 1 or $\overline{1}$ simple if it is removed with a type (1) move and is involved in no other moves. Let $b(j)$ be the number of strictly decreasing chains in $\left[(1), \omega_{n}\right]$ in which the $j^{\text {th }} 1$, counting from left to right, is the rightmost 1 that is not simple. Then

$$
\mu_{\mathcal{C}^{(m)}}\left((1), \omega_{n}\right)=b(1)+b(2)+\cdots+b(n) .
$$

Note that $b(j)$ does not depend on $n$ (as long as $n \geq j$ ) since if the $j^{\text {th }} 1$ is the rightmost non-simple part, any decreasing chain must begin in only one way: by removing, from right to left and with type (1) moves, all the parts to the right of the $j^{\text {th }} 1$.

We will use induction to show that $b(j)=2^{j-1}$ for any $j \leq n$, from which the proposition follows. Clearly $b(1)=1$. Now suppose $j>1$ and the $j^{\text {th }} 1$ is the rightmost non-simple 1. There are two types of decreasing chains to consider. First, all 1's and 1 's except the $j^{\text {th }} 1$ can be simple. There is one chain of this type (the labels are included above the arrows):

$$
\cdots \stackrel{(2 j, 1)}{\longrightarrow}(1, \overline{1}, \ldots, 1, \overline{1}, 1) \stackrel{(2 j-2,1)}{\longrightarrow}(1, \overline{1}, \ldots, 1,1) \stackrel{(2 j-3,1)}{\longrightarrow} \cdots \stackrel{(2,1)}{\longrightarrow}(1,1) \stackrel{(1,1)}{\longrightarrow}(1) .
$$

In the second type of chain, there is an index $i<j$ such that all 1's and $\overline{1}$ 's to the right of the $i^{\text {th }} \overline{1}$ are simple (except for the $j^{\text {th }} 1$ ) and removed from right to left with type (1) moves. Then a type (2) move is made involving the $i^{\text {th }} 1$, the $i^{\text {th }} \overline{1}$, and the $j^{\text {th }} 1$, followed by a type (1) move in the same urn:

$$
\begin{aligned}
\cdots \stackrel{(2 j, 1)}{\longrightarrow}(1, \overline{1}, \ldots, 1, \overline{1}, 1) \stackrel{(2 j-2,1)}{\longrightarrow}(1, \overline{1}, \ldots, 1,1) \stackrel{(2 j-3,1)}{\longrightarrow} \cdots \\
\quad \stackrel{(2 i+1,1)}{\longrightarrow}(1, \overline{1}, \ldots, 1, \overline{1}, 1) \stackrel{(2 i, 2)}{\longrightarrow}(1, \overline{1}, \ldots, 2) \stackrel{(2 i, 1)}{\longrightarrow}(1, \overline{1}, \ldots, 1)=\omega_{i} \longrightarrow \cdots
\end{aligned}
$$

Another type (1) move in the $2 i^{\text {th }}$ urn cannot be made to continue this decreasing chain. Therefore this chain can be continued with only the $b(i)$ chains in which the $i^{\text {th }} 1$ is nonsimple. We have shown that $b(j)=1+b(1)+b(2)+\cdots+b(j-1)$ for $j>1$, so by induction $b(j)=2^{j-1}$ and the proof is complete. 
It is possible to adapt this proof to give a bijection between nonempty subsets of $[n]$ and decreasing chains, by considering the set of non-simple 1's. We leave such a proof to the reader.

We now consider a "truncated" version of $\mathcal{C}^{(m)}$. Define the poset $\mathcal{C}_{n}^{(m)}$ by

$$
\mathcal{C}_{n}^{(m)}:=\widehat{1} \cup \bigcup_{1 \leq i \leq n} \operatorname{Comp}^{(m)}(i)
$$

with the order relation as before except with a new maximal element $\widehat{1}$ that covers all the compositions in $\mathrm{Comp}^{(m)}(n)$.

Corollary 4.4. The poset $\mathcal{C}_{n}^{(m)}$ is shellable, with Möbius function

$$
\mu(\emptyset, \widehat{1})=(-1)^{n+1}(m-1)^{n} .
$$

Proof. First we want to show that every $m$-colored composition $\alpha=\left(\varepsilon_{1} \alpha_{1}, \varepsilon_{2} \alpha_{2}, \ldots, \varepsilon_{k} \alpha_{k}\right)$ of at most $n$ lies below the composition $\gamma^{n} \models_{m} m n$, defined as the concatenation of $n$ copies of $\gamma=\left(1, \omega 1, \omega^{2} 1, \ldots, \omega^{m-1} 1\right)$. To the $i^{\text {th }}$ part of $\alpha$ we can associate $\alpha_{i}$ copies of $\gamma$. First, we use covering relations of type (2) (as originally described), $\alpha_{i}$ times to split the part into all parts of size 1 and color $\varepsilon_{i}$. Then we use covering relations of type (3) to fill in the remaining 1's of different colors. Therefore $\mathcal{C}_{n}^{(m)}$ is obtained via rank selection from the interval $\left[\emptyset, \gamma^{n}\right]$, so shellability follows by results of $[1]$.

For the Möbius function:

$$
\begin{aligned}
\mu(\emptyset, \widehat{1})=-\sum_{|\alpha| \leq n} \mu(\emptyset, \alpha) & =-\left(1+\sum_{k=1}^{n}(-1)^{k} m(m-1)^{k-1}\right) \\
& =-\left(1-m \sum_{k=0}^{n-1}(1-m)^{k}\right) \\
& =-\left(1-m\left(\frac{1-(1-m)^{n}}{1-(1-m)}\right)\right) \\
& =(-1)^{n+1}(m-1)^{n} .
\end{aligned}
$$

\section{Proof of CL-shellability}

In this section we give the proof of Theorem 4.1. We first note that the labeling is a well defined chain labeling. That is, if two chains agree on their first $k$ edges, then their first $k$ labels agree. This is clear from the definition.

The labeling of maximal chains in $[\emptyset, \alpha]$ gives an induced labeling on rooted intervals $([\beta, \alpha], c)$, where $c$ is a maximal chain in $[\emptyset, \beta]$. This induced labeling is of the same kind, 
so it suffices to check that the properties of a CL-labeling hold in an arbitrary interval $[\beta, \alpha]$.

First, we want to show that the chain with the lexicographically first label has a weakly increasing label. The lexicographically first label is well defined, since all the moves from a given distribution of balls in urns have distinct labels. Moreover, we can describe the lexicographically first chain, $c_{0}$, as follows. If

$$
c_{0}=\left(\alpha=\alpha^{0} \succ \alpha^{1} \succ \cdots \succ \alpha^{k}=\beta\right)
$$

then at each step, to move down from $\alpha^{r-1}$ to $\alpha^{r}$, we must remove a ball from an urn as far to the left as possible, such that the new composition is still in the interval $[\beta, \alpha]$. To prove that $\lambda_{1}\left(c_{0}\right) \leq \lambda_{2}\left(c_{0}\right) \leq \cdots \leq \lambda_{k}\left(c_{0}\right)$, we use the following lemma.

Lemma 5.1. On an interval of length two, the chain with the lexicographically first label is weakly increasing.

Proof. On an interval of length two, all chains correspond to removing two balls from urns, such that the starting and ending distributions are the same. For the chain $c_{0}$ with the lexicographically first label, the urns are consecutively chosen to be as far to the left as possible. Suppose that one ball is removed from urn $U_{i}$ and the other ball is removed from urn $U_{j}$.

If $j>i+1$, then there is a nonempty urn between $U_{i}$ and $U_{j}$, and removing a ball from one of the urns does not affect the possibility of removing the other ball from its urn. Therefore it is clear that $\lambda\left(c_{0}\right)$ is weakly increasing.

Suppose that $j=i+1$, so there is no urn between $U_{i}$ and $U_{j}$. Removing a ball from $U_{i}$ does not affect the possibility of removing a ball from $U_{i+1}$, unless $\varepsilon_{i} \neq \varepsilon_{i+1}=\varepsilon_{i-1}$ and $\alpha_{i}=1$. In this case, the urns could have been chosen to be $U_{h}$ and $U_{i}$, for an appropriate urn $U_{h}$ with $h<i$, making a type (1) or type (3) move in $U_{h}$ and then a type (2) move in urn $U_{i}$. However, this contradicts our assumption that $U_{i}$ was chosen to be the leftmost possible. So if $i \neq j, \lambda\left(c_{0}\right)$ is weakly increasing.

The only remaining case is if $U_{i}=U_{j}$. If $\alpha_{i}>2$, then we have the weakly increasing label $\lambda\left(c_{0}\right)=((i, 1),(i, 1))$. Now suppose that $\alpha_{i}=2$ and $\varepsilon_{i-1} \neq \varepsilon_{i}$. Then we also have $\lambda\left(c_{0}\right)=((i, 1),(i, 1))$. If $\varepsilon_{i-1}=\varepsilon_{i}$, then $c_{0}$ is found by choosing an appropriate urn $U_{h}, h<i$ and making a type (1) or (3) move in urn $U_{h}$ and then a type (1) move in urn $U_{i}$. Again, this contradicts our assumption that $U_{i}$ was chosen to be the leftmost possible. Therefore if $i=j, \lambda\left(c_{0}\right)$ is weakly increasing.

Returning to the general case, for every $r$, the induced labeling of $c_{0}$ on the chain $\alpha^{r-1} \succ \alpha^{r} \succ \alpha^{r+1}$ is lexicographically first on the interval $\left[\alpha^{r+1}, \alpha^{r-1}\right]$. Then by Lemma $5.1, \lambda_{1}\left(c_{0}\right) \leq \lambda_{2}\left(c_{0}\right) \leq \cdots \leq \lambda_{k}\left(c_{0}\right)$, i.e., $\lambda\left(c_{0}\right)$ is weakly increasing. Now it remains only to show no chain other than $c_{0}$ has a weakly increasing label.

If another chain results with the same distribution of balls into urns as the lexicographically first chain, including the locations of the empty urns, then the label on that chain must have a descent. To see this, consider the point where it deviates from the 
lexicographically first chain. It is leaving a ball behind in a lower numbered urn. At some later step it must remove a ball from that urn, which will create a descent.

Now we need to consider chains which result in the same distribution of balls into nonempty urns as the lexicographically first chain, but such that the empty urns are in different positions. Let $c$ be such a chain. Since the lexicographically first chain removes balls from urns from left to right, the final distribution of balls into urns for the lexicographically first chain has its nonempty urns as far to the right as possible.

Let $U_{1}, U_{2}, \ldots$ be the urns labeled from left to right. Let $U_{i}(c)$ be the number of balls in urn $U_{i}$ in the final distribution for the chain $c$. Let $r$ be the largest number such that $U_{r}(c)>0$ and $U_{r}\left(c_{0}\right)=0$. There is such an $r$ by our assumption on $c$. We must have a $j$ such that: 1) $U_{r+i}(c)=0$ for all $\left.1 \leq i \leq j, 2\right) U_{r+i}\left(c_{0}\right)=0$ for all $1 \leq i<j$, and 3) $U_{r+j}\left(c_{0}\right) \neq 0$. Note that the color and number of the balls in urn $U_{r}$ for $c$ is the same as the color and number of the balls in urn $U_{r+j}$ for $c_{0}$.

If $c$ has an increasing label, then the urns $U_{r+1}, \ldots, U_{r+j}$ must be emptied from left to right. Therefore at some point the urns $U_{r+1}, \ldots, U_{r+j-1}$ are empty and we need to remove the last ball from $U_{r+j}$. The only way to do this is to use a move of type $(2)$, which has a label $(r, 2)$ and creates a descent.

\section{References}

[1] A. Björner, Shellable and Cohen-Macaulay posets, Trans. Amer. Math. Soc. 260 (1980), 159-183.

[2] A. Björner, The Möbius function of subword order, Invariant Theory and Tableaux (ed. D. Stanton), IMA Volumes in Math. and Applic., Vol. 19, Springer-Verlag, New York, 1990, pp. 118-124.

[3] A. Björner, A. Garsia and R. Stanley, Cohen-Macaulay partially ordered sets in Ordered Sets, (I. Rival, ed.), Reidel, Dordrecht/Boston, 1982, pp. 583-615.

[4] S. Fomin, Duality of Graded Graphs, J. Algebraic Combinatorics 3 (1994), 357-404.

[5] D. Krob and J. Thibon, Noncommutative symmetric functions IV: Quantum linear groups and Hecke algebras at $q=0$, J. Algebraic Combinatorics 6 (1997), 339-376.

[6] S. Poirier, Cycle type and descent set in wreath products, Discrete Mathematics 180 (1998), 315-343.

[7] R. Stanley, Enumerative Combinatorics, Volume II, Cambridge University Press, 2001. 